\title{
A global optimization strategy for 3D-2D registration of vascular images
}

\author{
K. K. Lau and Albert C. S. Chung \\ Lo Kwee-Seong Medical Image Analysis Laboratory, \\ Department of Computer Science and Engineering, \\ The Hong Kong University of Science and Technology, Hong Kong. \\ achung@cse.ust.hk
}

\begin{abstract}
Although the presence of local minima is one of the major problems in high-dimensional image registration, only a few experimental works have been carried out to address this problem. In this study, a 3D-2D vascular image feature-based registration is done by producing Digital Reconstructed Radiographs (DRRs) of 3D images to match against the target 2D images. In addition, we propose a global optimization method based on the use of Powell's method at different resolution levels. To search the global minimum as effectively as possible, a large set of sample test points are systematically generated. The values of dissimilarity to the registered images in lower resolution environment are calculated. Powell's method is then applied to those test points with the lowest values for further minimization in the higher resolution. It is experimentally shown that our method can identify the global optimum in a normal clinical setting. The findings can have potential usage in the reconstruction of 3D models (e.g. guide wires) based on 2D medical visual information.
\end{abstract}

\section{Introduction}

The determination of the position and orientation of a 3D model in relation to 2D images is a crucial and fundamental computer vision problem [1], which has potential applications in a number of different medical areas. During the endovascular treatment of intracranial aneurysms, for example, a radiologist relies on 2D projection images (e.g. Digital subtraction Angiogram) to visualize the operation.

Often, a preoperative 3D image (e.g. 3DRA) of the vasculature is produced for the radiologist to familiar with the structure beforehand. A mental reconstruction of the vasculature and its relative position and orientation to the operation tools is required; that can be a demanding task during operation [2]. It is proposed that by proper registering the preoperative 3D volumetric dataset to the real-time 2D images, 
a 3D model of the real-time scene can be computed and reconstructed, which will help radiologists to carry out the treatments.

The success of image registration depends on the global optimization of the cost function that quantifies the quality of alignment between the images being registered. In 3D-2D registration of vascular images, the existence of numerous local minima makes it impossible to locate the global solution by just applying purely mathematical optimization methods, such as Powell's method, simplex methods, gradient descent, etc., without a global optimization strategy. Although there are some global optimization methods (e.g. Genetic algorithm [3], Evolution strategy, etc.) developed, they cannot usually be applied to 3D image registration due to high computational expenses; few experimental works have been carried out to address this problem so far [4].

Unlike other image registration problems, local minimum is particularly problematic in vascular 2D-3D image registration. While we cannot find relevant information in the literature specifically discussed this problem, our past probing experiments showed that the effective searching ranges of minima are relatively narrow (in terms of physical distance in millimetre) in vascular images, compared to other medical images, such as brain T1 images. This is perhaps due to the rather irregular shapes of blood vessels. For this reason, it is extremely difficult, if possible, to assure the identification of global minima during registration.

In this study, we propose a practical and effective global optimization strategy to overcome this problem. The aim of this study is to help to develop a system that may register a 3D dataset, within a restricted transformation range, to a 2D image in a fully automatic manner. The central idea is to apply a systematic multi-start-point approach combined with a local optimization method (Powell's method) and a multi-resolution approach. Although images may be registered automatically with only multi-resolution approach [5], it may not be usually true in 3D registration. This fact will be demonstrated in the experiment in this study. The long term goal of using this study is to make the optimization strategy applicable in a full clinical setting.

\section{Methods}

\subsection{The Registration Algorithm}

Since it is much more computational expensive to use conventional image-based 2D-3D registration [6], we adopted a feature-based registration method in the expense of some accuracy. For vascular registration, usually, the centrelines of the blood vessels are used as the main feature. Among two relatively popularly referred methods, the registration method of Chan et al. [7] is selected for its inclusion of vessel thicknesses and its larger range of rotation than those in Yasuyo et. al's method [1]. Chan et al. showed that for the demonstrated cases their algorithm can be applied to registration between 3D magnetic resonance angiographic images and 2D digital subtraction angiographic images up to the required accuracy.

In this registration approach, vessels are first segmented by using global thresholding method. By using the extracted skeleton, the original binary volume can be represented and reconstructed by the set of spheres with the centres set to the 
coordinates of skeleton points, and radii equal to their distance transform values, which represent distance to the closest boundary. Given the rigid transformation matrix and the projective geometry, the centre of each sphere is projected to the projection plane. The length of its corresponding radius in the projection plane can be calculated based on projective geometry. Figure 1 illustrates the above preparation stage of the registration by showing the various steps involved.

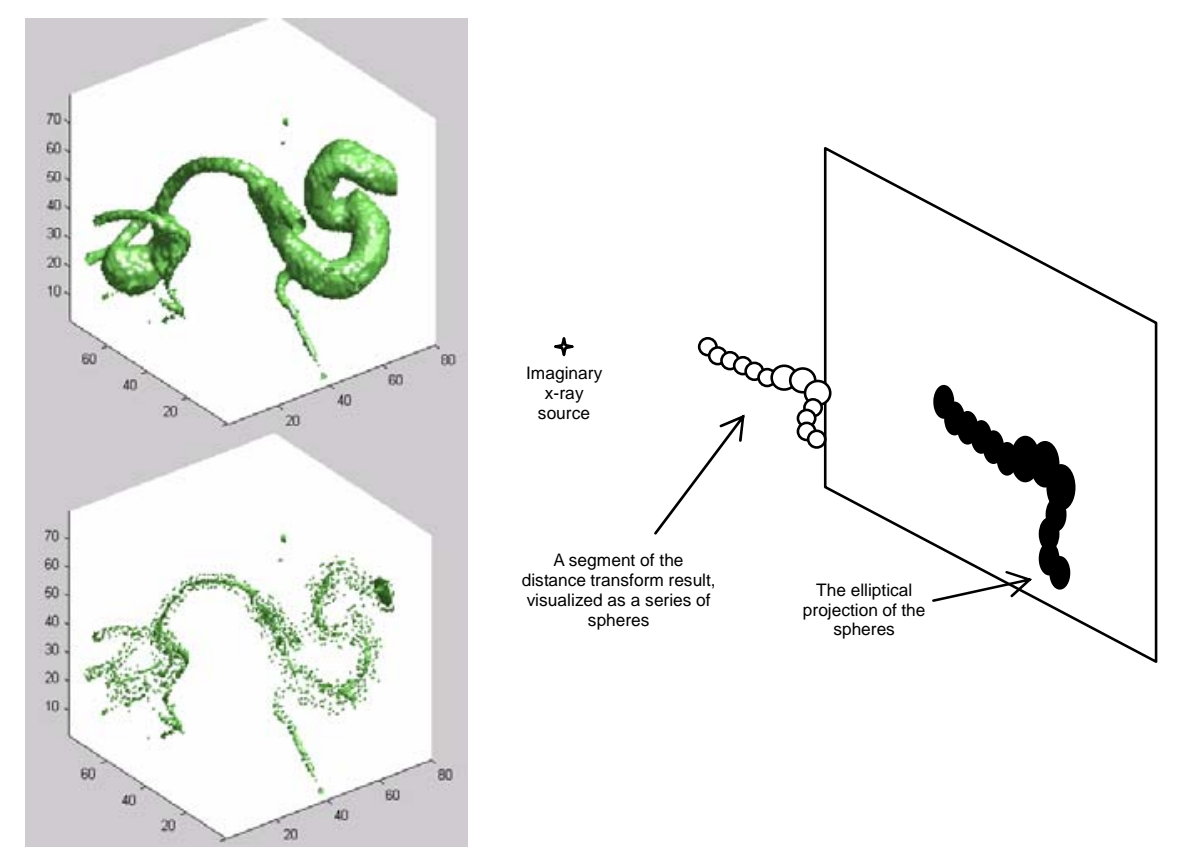

Figure 1: The vascular structure of the volumetric dataset after global threshold (top left). The positions in the volume containing distance value after the distance transform (bottom left). An illustrative diagram showing the reconstruction of a segment of the vascular structure based on the distance transform value and the projection of the reconstructed structure (right).

The sum of squared differences, SSD, is employed as the cost function to measure the difference between the projected 2D image from the 3D dataset and the targeted 2D posture. The SSD between the two images is given by:

$$
\operatorname{SSD}\left(I_{1}, I_{2}\right)=\sum_{i \in \phi}\left(I_{1}(i)-I_{2}(i)\right)^{2},
$$

where i and $\phi$ donate the pixel locations and overlapping between the images. The value of SSD is expected to be minimal, when the two images are precisely registered. The goal of the registration is to find the transformation $\mathbf{T}$ that gives the minimum SSD.

$$
\mathbf{T}=\arg \min _{T} S S D\left(I_{1}, I_{2}(T)\right),
$$

where $I_{1}$ is the image to be registered and $I_{2}$ is the projected image of the $3 D$ 
volumetric dataset.

With this SSD cost function, Powell's method is used to search for the adjacent local minimum at any given initial point. In this study, the Powell's method is used together with annealing setting for progressively searching of minima.

To verify the practicality of registration algorithm replicated in the current study on the real clinical data, we obtained one set of the image data for examination. The image data consisted of a 3D Phase Contrast (PC) MRA and a DSA (2D) to be aligned with each other. A segment of the 3D data was obtained by trilinear interpolation, followed by global thresholding and region of interest selection. It was found that the projection of the segment can be aligned with the DSA image using the registration algorithm (Figure 2). Nevertheless, since some critical information of the datasets, such as ground truth and the focal length (the distance between the $\mathrm{x}$-ray source and the projection frame), was not known, precise error estimation may not be obtained.
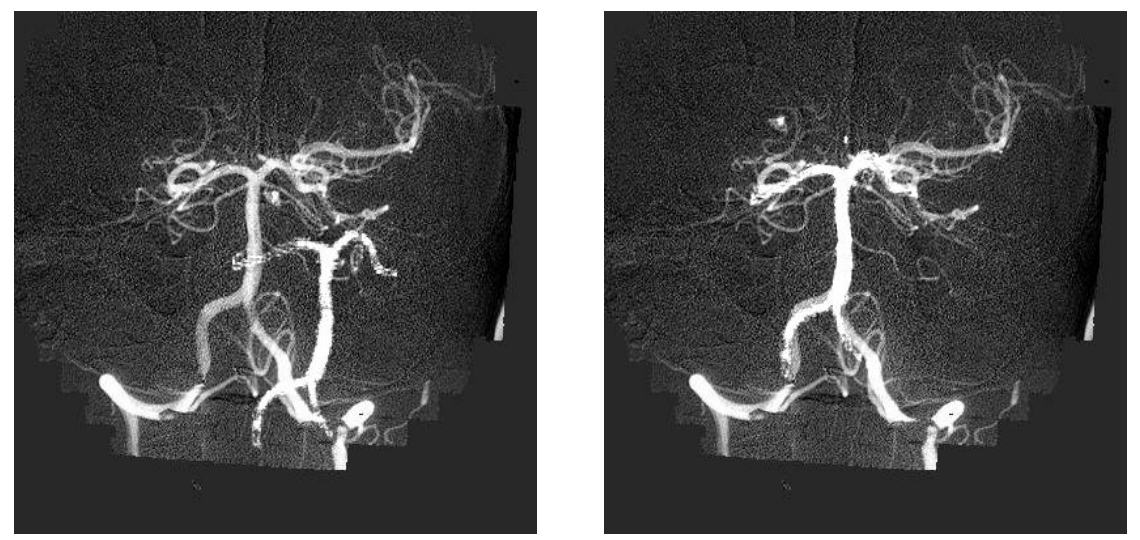

Figure 2: The projection of the 3D MRA segment projected on the DSA image before (left) and after (right) registration.

\subsection{The Optimization Strategy}

This strategy applies a systematic multi-resolution initial search and Powell's method for optimization. Jenkinson \& Smith [8] have developed a strategy for 3D-3D brain image registration. The current strategy is structurally simpler than theirs and is applied to 3D-2D vascular image registration. Although 3D-2D registration requires the generation of Digital Reconstructed Radiograph (DRR) and is usually more computational expensive than 3D-3D registration, with the current structurally simpler strategy, it is possible to complete an optimization within a reasonable time.

Phase 1: Resolution reduction. To search for the global minimum, first, a lower resolution version of the volumetric dataset is created by averaging each $2 \times 2 \times 2$ voxel sets followed by smoothing using a low-pass filter. 
Phase 2: Generation of the initial test sets. A 3D-2D registration involves the transformation of a 3D dataset, and so it is a six dimension transformation problem with 3 dimension of translation and 3 dimension of rotation (X, Y, Z, Rx, Ry and Rz). A test set consists of a value in each of the six dimensions. For each of the six dimensions, a series of values evenly distributed within the range of interest is used. For example, if the range in $\mathrm{X}$ dimension is from $-15 \mathrm{~mm}$ to $+15 \mathrm{~mm}$, the series of values within this range can be $-10 \mathrm{~mm}, 0 \mathrm{~mm}$ and $10 \mathrm{~mm}$. With such method, a value in each dimension is selected in turn, until all possible combinations are used. Thus, the total number of initial test sets is equal to the product of the number of values used in all the six dimensions.

Phase 3: Initial search. The outputs of the cost function generated using the initial test sets were computed in the low resolution environment. The test sets with the $n$ lowest cost function outputs are identified.

Phase 4: Multi-resolution optimization. Optimization using Powell's method is run for these identified test sets in the low resolution environment. These results are compared and the test set with the best optimization result is identified for further optimization in the higher resolution environment to obtain the final solution.

\subsection{The Datasets}

In this study, two clinical 3D-RA datasets acquired by a Philips Integris imager were used. The data volumes are $256 \times 256 \times 256$ voxels with a voxel size of $0.188 \times$ $0.188 \times 0.188 \mathrm{~mm}^{3}$. The volumes were cropped to include only the region of interests. To facilitate the registration process to an acceptable level, the resolution was reduced and smoothed. The final volume used for registration is $80 \times 80 \times 80$ voxels with the new voxel size of $0.376 \times 0.376 \times 0.376 \mathrm{~mm}^{3}$.

For the 2D images to be registered, synthetic 2D images, instead of 2D images from a different modality, were used for testing the optimization strategy. These images were projected from 3D datasets at known orientations and positions. There are two advantages of using synthesis 2D images. First, by doing so, the global solution will be precisely known without on-the-site calibration. Second, other sources of error irrelevant to the registration process can be eliminated.

\subsection{The Experiment}

In this study, the range of interest for each dimension has been restricted to $\pm 37 \mathrm{~mm}$ for translational dimension and $\pm 15^{\circ}$ for each rotation dimension. Although the translational range is smaller than that in typical clinical practise [1], it is nevertheless completely feasible for the professional to restrict patients' movement to this range without using complex calibration tools.

In principle, the translational range may be increased without the lost of reliability, it is restricted to the range based mainly on efficiency consideration. For each rotational dimension, $-10^{\circ}, 0^{\circ}, 10^{\circ}$ were selected as used values. For in-plane translation dimensions ( $\mathrm{X}$ and $\mathrm{Y}$ ), $-30 \mathrm{~mm},-22.5 \mathrm{~mm},-15 \mathrm{~mm},-7.5 \mathrm{~mm}, 0 \mathrm{~mm}, 7.5 \mathrm{~mm}$, $15 \mathrm{~mm}, 22.5 \mathrm{~mm}$, and $30 \mathrm{~mm}$ were selected. For $Z$ plane, since the effect of movement to the similarity value is much lower, for the sake of efficiency, a sparse 
scale, $-22.5 \mathrm{~mm}, 0 \mathrm{~mm}$ and $22.5 \mathrm{~mm}$, is used. With this setting, there are $(3 \times 3 \times 3 \times 9$ x 9 x 3) 6561 samples of test sets.

To test the registration algorithm and the optimization strategy, 10 target 2D images to be registered were randomly generated with the orientation parameters chosen within the above allowable range. The registration algorithm with only Powell's method (without any optimization strategy) was also used to register with the 10 generated postures for performance comparison. The starting positions for these registrations were selected randomly with the orientation parameters chosen within the above allowable range. Figure 3 shows two examples of the registration experiment; the images in the middle column are the generated 2D images to be registered, while the images on the right column are the random starting positions.

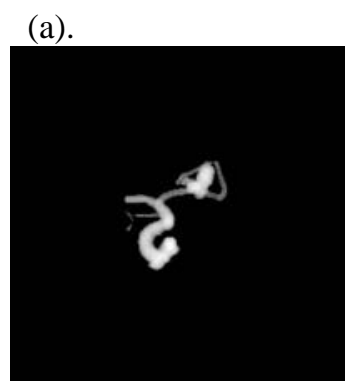

(d).

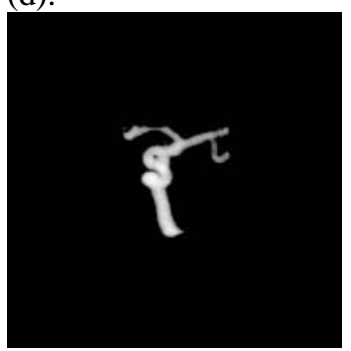

(b).

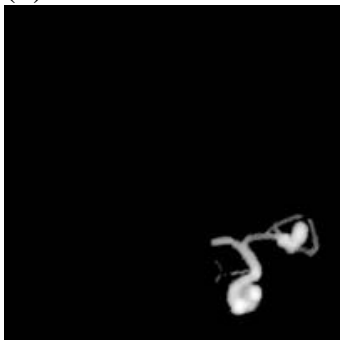

(e).

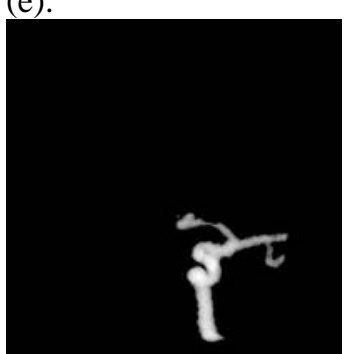

(c).

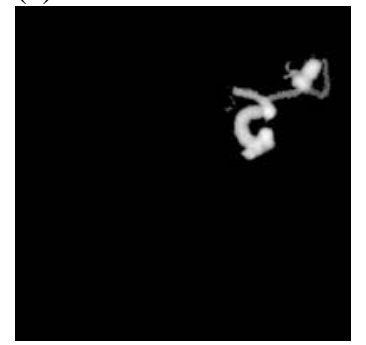

(f).

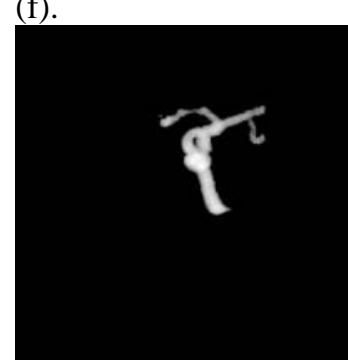

Figure 3: The projected image while the 3D dataset at the zero position $\left(0^{\circ}, 0^{\circ}, 0^{\circ}, 0\right.$, $0,0)$ of the Patient $1(\mathrm{a})$, one of the generated 2D images (while the 3D dataset at $\left.\left(-10.5^{\circ}, 5.9^{\circ},-3.7^{\circ}, 27.0,26.5,7.0\right)\right)$ to be registered (b), and the image at one of the randomly initial positions, (while the 3D dataset at $\left(13.0^{\circ},-7.0^{\circ},-10.2^{\circ}, 28.0,-19.7\right.$, 10.9)) at which the searches start (c); for the Patient 2 , the projected image while the $3 \mathrm{D}$ dataset at the zero position (d), one of the generated 2D images (while the 3D dataset at $\left.\left(10.4^{\circ}, 0.8^{\circ},-8.9^{\circ}, 12.9,25.4,-36.0\right)\right)$ to be registered (e), and the image at one of the randomly initial positions, (while the 3D dataset at $\left(-11.0^{\circ},-8.8^{\circ}, 3.2^{\circ}, 9.7\right.$, -9.7, 5.6)) at which the searches start (f).

Six registrations starting from different positions were carried out in the higher resolution environment for each of the ten generated postures. For further comparison, in a similar way as above, the registration algorithm with only Powell's method was done first in the low resolution environment; the results were further optimized in the higher resolution environment with Powell's method. Each time when Powell's method is applied in this study, 15 complete cycles of iterations were 
used; the searching would stop upon the finishing of these 15 iterations.

\section{Results}

(a).

\begin{tabular}{|c|c|c|c|}
\hline $\begin{array}{l}\text { Random } \\
\text { Postures }\end{array}$ & $\begin{array}{l}\text { Global } \\
\text { optimization } \\
\text { strategy }\end{array}$ & $\begin{array}{l}\text { Powell's method only } \\
\text { in higher resolution } \\
\text { environment }\end{array}$ & $\begin{array}{l}\text { Powell's method in } \\
\text { multi-resolution } \\
\text { environment }\end{array}$ \\
\hline 1 & M & $\mathrm{F}, \mathrm{F}, \mathrm{F}, \mathrm{M}, \mathrm{F}, \mathrm{F}$ & $F, F, F, F, F, F$ \\
\hline 2 & $\mathrm{~S}$ & $\mathrm{~F}, \mathrm{~F}, \mathrm{~F}, \mathrm{~F}, \mathrm{~F}, \mathrm{~F}$ & F, F, F, F, F, F \\
\hline 3 & M & F, F, F, F, F, F & F, F, F, F, F, F \\
\hline 4 & M & F, M, F, F, F, F & F, M, F, F, F, F \\
\hline 5 & $\mathrm{~S}$ & $\mathrm{~F}, \mathrm{~F}, \mathrm{~F}, \mathrm{~F}, \mathrm{M}, \mathrm{F}$ & F, F, F, F, S, F \\
\hline 6 & $S$ & F, F, F, F, M, M & $\mathrm{F}, \mathrm{F}, \mathrm{F}, \mathrm{F}, \mathrm{S}, \mathrm{M}$ \\
\hline 7 & $\mathrm{~S}$ & F, F, F, F, F, F & $\mathrm{F}, \mathrm{F}, \mathrm{F}, \mathrm{F}, \mathrm{F}, \mathrm{F}$ \\
\hline 8 & M & $\mathrm{F}, \mathrm{F}, \mathrm{F}, \mathrm{F}, \mathrm{F}, \mathrm{F}$ & $\mathrm{M}, \mathrm{F}, \mathrm{F}, \mathrm{F}, \mathrm{F}, \mathrm{F}$ \\
\hline 9 & $\mathrm{~S}$ & $\mathrm{~F}, \mathrm{~F}, \mathrm{~S}, \mathrm{~F}, \mathrm{~F}, \mathrm{~F}$ & $\mathrm{~F}, \mathrm{~F}, \mathrm{~S}, \mathrm{~F}, \mathrm{~F}, \mathrm{~F}$ \\
\hline 10 & S & F, F, F, F, F, M & F, F, F, F, F, S \\
\hline Overall & $60.0 \%$ & $1.7 \%$ & $6.7 \%$ \\
\hline \multirow{2}{*}{ Rates } & $40.0 \%$ & $10.0 \%$ & $5.0 \%$ \\
\hline & $0.0 \%$ & $88.3 \%$ & $88.3 \%$ \\
\hline
\end{tabular}

(b).

\begin{tabular}{|c|c|c|c|}
\hline $\begin{array}{l}\text { Random } \\
\text { Postures }\end{array}$ & $\begin{array}{l}\text { Global } \\
\text { optimization } \\
\text { strategy }\end{array}$ & $\begin{array}{l}\text { Powell's method only } \\
\text { in higher resolution } \\
\text { environment }\end{array}$ & $\begin{array}{l}\text { Powell's method in } \\
\text { multi-resolution } \\
\text { environment }\end{array}$ \\
\hline 1 & M & F, F, F, F, F, F & $\mathrm{M}, \mathrm{M}, \mathrm{M}, \mathrm{F}, \mathrm{F}, \mathrm{F}$ \\
\hline 2 & $\mathrm{~S}$ & F, M, F, F, F, F & F, S, M, F, F, F \\
\hline 3 & M & F, S, F, F, F, F & F, M, F, F, F, F \\
\hline 4 & $\mathrm{~F}$ & $F, F, F, F, M, F$ & $\mathrm{~F}, \mathrm{~F}, \mathrm{~F}, \mathrm{~F}, \mathrm{~F}, \mathrm{~F}$ \\
\hline 5 & S & $\mathrm{F}, \mathrm{F}, \mathrm{F}, \mathrm{F}, \mathrm{F}, \mathrm{F}$ & $F, F, F, F, F, F$ \\
\hline 6 & $\mathrm{~F}$ & $F, F, F, F, F, F$ & $F, F, F, F, F, F$ \\
\hline 7 & M & $F, F, F, F, F, F$ & $\mathrm{M}, \mathrm{F}, \mathrm{F}, \mathrm{F}, \mathrm{F}, \mathrm{F}$ \\
\hline 8 & S & $\mathrm{F}, \mathrm{F}, \mathrm{M}, \mathrm{F}, \mathrm{F}, \mathrm{F}$ & $\mathrm{F}, \mathrm{F}, \mathrm{S}, \mathrm{M}, \mathrm{S}, \mathrm{F}$ \\
\hline 9 & $\mathrm{~S}$ & $F, F, F, F, F, S$ & $\mathrm{~F}, \mathrm{~F}, \mathrm{~F}, \mathrm{~F}, \mathrm{~F}, \mathrm{~S}$ \\
\hline 10 & M & F, F, F, F, F, F & F, F, F, F, F, F \\
\hline Overall & $40.0 \%$ & $3.3 \%$ & $6.7 \%$ \\
\hline \multirow[t]{2}{*}{ Rates } & $40.0 \%$ & $5.0 \%$ & $11.7 \%$ \\
\hline & $20.0 \%$ & $91.7 \%$ & $81.6 \%$ \\
\hline
\end{tabular}

Table 1: The results of the three optimization methods: (a) and (b) show the results for data of Patient 1 and Patient 2 respectively. (F- Failed; M- Marginal; SSuccessful.) 
The results are shown in Table 1 . The three columns show respectively the results using the three different optimization methods described above. The results shown were based on the calculation of registration error. The target registration error in the $3 \mathrm{D}$ space instead of projection error on the projection plane was used to evaluate the registration quality, since the former measure affects the surgery most directly. The eight vertexes of the volumetric data are selected as the gold standard registration points. The mean target registration errors, mTREs, were calculated by Kraats et. al's method [8]:

$$
\operatorname{mTRE}\left(P, T_{\text {reg }}, T_{\text {gold }}\right)=\frac{1}{k} \sum_{i=1}^{k}\left\|T_{\text {reg }} p_{i}-T_{\text {gold }} p_{i}\right\|,
$$

where $P$ is the set of considered points, $T_{\text {gold }}$ is the known transformation, $T_{\text {reg }} P_{i}$ is the position of the point $i$ after the transformation based on the registration result. In the current case, $\mathrm{k}$ is eight.

For simplicity of discussion, the error rates were classified into three categories only. In Table 1, the letter S represents the registration being successful with the registration error within an acceptable range of $3 \mathrm{~mm}$ [6]. The letter $\mathrm{M}$ represents the registration was not completely successful with the optimization results being close to the ground truth with the registration error within a few times of the acceptable range (12mm was used for cut-off value). The letter F represents the registration being failed with the optimization results far from the optimal solution.

It is found that with the global optimization strategy, six of them returned the global minimum and the remaining four reached the region adjacent to the global minimum for Patient 1, and four of them returned the global minimum, four of them reached adjacent region and two of them failed for Patient 2. For the other two methods, only about $8 \%-18 \%$ of the registrations reached S or M level.

\section{Discussion and Conclusion}

This study has demonstrated a feasible global optimization strategy for 3D-2D vascular image registration. It is found that the successful rate of identifying the global minima is much higher than that of the proposed strategy.

Without a reliable global optimization strategy, the registration process would not be completely automatic, no matter how high quality is the registration method itself. Although, up to this developmental stage, the strategy cannot guarantee the successful identification of the global minima, the study moves a step toward this goal. In order to achieve the goal, a few problems ahead still needed to be solved to improve the optimization strategy.

First, failure of the strategy has to be prevented as far as possible. The two failed cases of Patient 2 were furthered analysed. It was found that in one case, the searching was stopping as a region that slightly away from the adjacent region; this will be discussed together in the next paragraph. In the other case, the solution was rather different from the optimal solution; this problem may be prevented by using a slightly denser scale in the initial sampling at the expense of efficiency, but its trade-off and the benefit have to be examined extensively in further analysis. 
Second, the stopping at the adjacent region near the optimal solution need to be prevented. It is surprising that the registration process unable to return to the goal at the surrounding of the global minima. There are two possible reasons for explaining this stopping.

The first possibility is that the roughness of the original clinical data may create local minimum close to the global minimum. The second possibility is that the optimization process is exception slow that it is unable to reach the global minimum within a reasonable time. Further work, such as re-analysis processing of raw data, may be carried out to overcome this stopping problem.

Finally, in order to make the optimization a real-time process, the time required to complete an optimization need to be greatly reduced. The current global optimization process took about 13 minutes with a $2.5 \mathrm{GHz}$ processor (512M RAM) simulated on a Matlab platform. With a high-end computer and lower level computer language (such as $\mathrm{C}++$ ) and further optimization of the searching algorithm, it is possible to make the process down to below half minute.

It is known that the global optimization is required only once in the whole process, since subsequent movement can be tracked relatively easily based on the first successful registration.

One issue worth noticing here is that it has been argued that multi-resolution approach may help in identifying global minima. However, detailed analysis of the stopping positions seem to suggest that the multi-resolution approach would help to facilitate the searching to the adjacent local minima, rather than to transcend the local minima.

In the clinical setting, it is important to make the use of information as automatic as possible to alleviate the workload and mental pressure of the medical professional. We believe that the direction of this study may help the development of equipment that facilitates clinical operation. With the advancement of global optimization technique and the reconstruction techniques of surgical tools in endovascular treatment [2], it is possible that operation process can be visualized in 3D using only $2 \mathrm{D}$ real-time monitoring system.

\section{References}

[1] Yasuyo Kita, Dale L. Wilson, and Alison Noble. Real-time registration of 3D Celebral Vessels to X-ray Angiograms. MICCAI 1998, p1125 - 1133.

[2] Shirley A. M. Baert, Graeme P. Penney, Theo van Walsum, and Wiro J. Niessen. Precalibration Versus 2D-3D Registration for 3D Guide Wire Display in Endovascular Interventions. MICCAI 2004, p. 577 - 584.

[3] Rongkai Zhao, Geneva G. Belford, and Michael Gabriel. A cluster-assisted global optimization method for high resolution medical image registration. The 6th IEEE Southwest Symposium, p. 66 - 70.

[4] Mark Jenkinson, and Stephen Smith. A global optimisation method for robust affine registration of brain images. Medical Image Analysis 2001, p.143 - 156. 
[5] M. Corvi and G. Nicchiotti, Multiresolution Image Registration, Proceedings of IEEE International Conference on Image Processing 1995, p. 224 - 227.

[6] Robert A. McLaughlin, John Hipwell, David J. Hawkes, Ailson Noble, James V. Byrne, and Tim C. Cox. A Comparison of a Similarity-Based and a Feature-Based 2-D-3-D Registration Method for Neurointerventional Use. IEEE Transactions on Medical Imaging, 2005, p. 1058 - 1066.

[7] H. M. Chan, Albert C. S. Chung, Simon C. H. Yu and William M. Wells III. 2D-3D Vascular Registration between Digital Subtraction Angiographic (DSA) And Magnetic Resonance Angiographic (MRA) images. IEEE International Symposium on Biomedical Imaging 2004, p.708 - 711.

[8] Everine B. van de Kraats, Graeme P. Penney, Dejan Tomazevic, Theo van Walsum, Wiro J. Niessen, Standardized Evaluation Methodology for 2D-3D Registration, IEEE Transactions on Medical Imaging, 2005, vol. 24, p. 1177 1190.

[9] Lilla Zollei, 2D-3D rigid-body registration of X-ray Fluoroscopy and CT images, M.S. Thesis, Massachusetts Institute of Technology, 2001.

[10] Ho-Ming Chan. A supervised learning framework for multi-model rigid registration with applications to angiographic images, M.S. Thesis, Hong Kong University of Science and Technology, 2003.

[11] Graeme. P. Penney. Registration of Tomographic images to X-ray projections for use in image guided interventions. PhD Thesis, University of London, 1999.

[12] Nikhil Gagvani. Skeletons and Volume Thinning in Visualization, MS. thesis, Rutger University, New Brunswick, New Jersey, 1997. 